\title{
A New Fractional Model for Cancer Therapy with M1 Oncolytic Virus
}

\author{
Majda El Younoussi $\mathbb{D}^{1},{ }^{1}$ Zakaria Hajhouji, ${ }^{1}$ Khalid Hattaf $\mathbb{D}^{1,2}$ and Noura Yousfi ${ }^{1}{ }^{1}$ \\ ${ }^{1}$ Laboratory of Analysis, Modeling and Simulation (LAMS), Faculty of Sciences Ben M'sik, Hassan II University of Casablanca, \\ P.O Box 7955 Sidi Othman, Casablanca, Morocco \\ ${ }^{2}$ Centre Régional des Métiers de l'Education et de la Formation (CRMEF), Derb Ghalef, Casablanca 20340, Morocco
}

Correspondence should be addressed to Khalid Hattaf; k.hattaf@yahoo.fr

Received 18 April 2021; Revised 22 May 2021; Accepted 28 May 2021; Published 7 June 2021

Academic Editor: Constantin Udriste

Copyright (C) 2021 Majda El Younoussi et al. This is an open access article distributed under the Creative Commons Attribution License, which permits unrestricted use, distribution, and reproduction in any medium, provided the original work is properly cited.

\begin{abstract}
The aim of this work is to propose and analyze a new mathematical model formulated by fractional differential equations (FDEs) that describes the dynamics of oncolytic M1 virotherapy. The well-posedness of the proposed model is proved through existence, uniqueness, nonnegativity, and boundedness of solutions. Furthermore, we study all equilibrium points and conditions needed for their existence. We also analyze the global stability of these equilibrium points and investigate their instability conditions. Finally, we state some numerical simulations in order to exemplify our theoretical results.
\end{abstract}

\section{Introduction}

Cancer is a collection of related diseases where some of the body's cells divide continuously and spread into surrounding tissues. Cancer is caused by certain changes to genes. It can start almost anywhere in the human body. Old or damaged cells survive when they should die; new cells form when the body does not need them. These extra cells can divide continuously and may form tumors. A tumor becomes dangerous when it begins to form extensions to neighboring areas (metastasis) [1]. This is why it is important to detect cancer as early as possible in order to avoid this migration. Cancer treatment is adapted according to each situation. There are different cancer treatments used alone or in combination, such as surgery, radiotherapy, chemotherapy, hormone therapy, immunotherapy, and virotherapy. Virotherapy is one of the new therapies; it consists in using a virus after having reprogrammed it. This virus is called oncolytic virus. Oncolytic viruses infect and destroy cancer cells; they use the cell's genetic machinery to make copies of themselves and subsequently spread to surrounding uninfected cells [2].

According to a medical experiment, in vitro, in vivo, and ex vivo studies showed potent oncolytic efficacy and high tumor tropism of alphavirus M1, which is a naturally occurring and a selective oncolytic virus targeting zinc-finger antiviral protein (ZAP) deficient cancer cells [3]. To model the role of the M1 virus in oncolytic virotherapy, Wang et al. [4] proposed a nonlinear system governed by ordinary differential equations (ODEs) that describe the growth of normal cells, tumor cells, and the M1 virus with limited nutrients. Elaiw et al. [5] extended the model presented in [4] by including spatial effects and anti-tumor immune response mediated by cytotoxic T lymphocyte (CTL) cells. The results in [5] indicated that the immune response has a negative impact on oncolytic M1 virotherapy, and it reduced its efficiency.

On the other hand, all the above mathematical models neglected the memory effect by considering only integerorder derivatives. However, fractional-order derivative provides an excellent tool for describing memory and hereditary properties which exist in most biological systems. For instance, Cole [6] proved that the membranes of cells of the biological organism have fractional-order electrical conductance since the memory means that the system's response is dependent not only on the current state but on its complete history. Therefore, the classical integer-order 
derivative does not reflect this memory effect because it is a local operator, unlike the fractional derivative.

The main purpose of this study is to develop a mathematical model governed by fractional-order differential equations (FDEs) to study the effect of memory on the dynamics of oncolytic M1 virotherapy. So, the rest of the paper is outlined as follows: the next section is devoted to the formulation of the model, including the well-posedness and the existence of equilibria. Section 3 focuses on stability analysis. Section 4 deals with numerical simulations in order to illustrate our main analytical results. Finally, a brief conclusion is given in Section 5.

\section{Model Formulation and Preliminaries}

In this section, we propose the following FDE model:

$$
\left\{\begin{array}{l}
D^{\alpha} S(t)=A-d S(t)-\beta_{1} S(t) N(t)-\beta_{2} S(t) T(t), \\
D^{\alpha} N(t)=r_{1} \beta_{1} S(t) N(t)-\left(d+\varepsilon_{1}\right) N(t), \\
D^{\alpha} T(t)=r_{2} \beta_{2} S(t) T(t)-\left(d+\varepsilon_{2}\right) T(t)-\beta_{3} T(t) V(t), \\
D^{\alpha} V(t)=B+r_{3} \beta_{3} T(t) V(t)-\left(d+\varepsilon_{3}\right) V(t),
\end{array}\right.
$$

where $S(t), N(t), T(t)$, and $V(t)$ are the concentrations of nutrient, normal cells, tumor cells, and $M_{1}$ virus at time $t$, respectively. The parameters $A$ and $B$ are the recruitment rates of nutrient and $M_{1}$ virus, respectively. Also, $B$ represents the minimum effective dosage of medication. The normal and tumor cells consume the nutrient at rates $\beta_{1} S N$ and $\beta_{2} S T$, respectively. The growth rate of normal cells as a result of consuming the nutrient is given by $r_{1} \beta_{1} S N$, while the growth rate of tumor cells is given by $r_{2} \beta_{2} S T$. The virus infects and kills tumor cells at rate $\beta_{3} T V$, and it replicates at rate $r_{3} \beta_{3} T V$. The parameter $d$ is the washout constant rate of nutrient and bacteria. The parameters $\varepsilon_{1}, \varepsilon_{2}$, and $\varepsilon_{3}$ are the natural death rates of normal cells, tumor cells, and $M_{1}$ virus, respectively. The operator $D^{\alpha}$ denotes the Caputo fractional derivative with $\alpha \in(0,1]$ that describes the memory effect.

It is important to note that the ODE mathematical model verifying potent oncolytic efficacy of M1 virus [4] is a special case of our model presented by system (1), and it suffices to take $\alpha=1$. Furthermore, to prove that our model is biologically well-posed, we assume that the initial conditions of (1) satisfy:

$$
\begin{aligned}
S(0) & =\phi_{1}(0) \geq 0, N(0)=\phi_{2}(0) \geq 0, T(0)=\phi_{3}(0) \\
& \geq 0, V(0)=\phi_{4}(0) \geq 0 .
\end{aligned}
$$

Theorem 1. If the initial conditions (2) are given, then there exists a unique solution of system (1) defined on $[0,+\infty)$. Moreover, this solution remains nonnegative and bounded for all $t \geq 0$.

Proof. It is not hard to show that the vector function of system (1) satisfies the first condition of Lemma 4 in [7]. It remains to prove the second condition. Let

$$
\begin{gathered}
X(t)=\left(\begin{array}{c}
S(t) \\
N(t) \\
T(t) \\
V(t)
\end{array}\right), \\
Y=\left(\begin{array}{c}
A \\
0 \\
0 \\
B
\end{array}\right) .
\end{gathered}
$$

Then

$$
D^{\alpha} X(t)=Y+A_{1} X(t)+A_{2} S(t) X(t)+A_{3} T(t) X(t),
$$

where

$$
\begin{aligned}
& A_{1}=\left(\begin{array}{cccc}
-d & 0 & 0 & 0 \\
0 & -\left(d+\varepsilon_{1}\right) & 0 & 0 \\
0 & 0 & -\left(d+\varepsilon_{2}\right) & 0 \\
0 & 0 & 0 & -\left(d+\varepsilon_{3}\right)
\end{array}\right), \\
& A_{2}=\left(\begin{array}{cccc}
0 & -\beta_{1} & -\beta_{2} & 0 \\
0 & r_{1} \beta_{1} & 0 & 0 \\
0 & 0 & r_{2} \beta_{2} & 0 \\
0 & 0 & 0 & 0
\end{array}\right), \\
& A_{3}=\left(\begin{array}{cccc}
0 & 0 & 0 & 0 \\
0 & 0 & 0 & 0 \\
0 & 0 & 0 & -\beta_{3} \\
0 & 0 & 0 & r_{3} \beta_{3}
\end{array}\right) .
\end{aligned}
$$

Thus,

$$
\left\|D^{\alpha} X(t)\right\| \leq\|Y\|+\left(\left\|A_{1}\right\|+\left\|A_{2}\right\|\|S\|+\left\|A_{3}\right\|\|T\|\right)\|X\| .
$$

This implies that the second condition of Lemma 4 in [7] is satisfied. Then system (1) has a unique solution on $[0,+\infty)$.

On the other hand and according to (1), we have

$$
\begin{aligned}
\left.D^{\alpha} S(t)\right|_{S=0} & =A>0, \\
\left.D^{\alpha} N(t)\right|_{N=0} & =0 \geq 0, \\
\left.D^{\alpha} T(t)\right|_{T=0} & =0 \geq 0, \\
\left.D^{\alpha} V(t)\right|_{V=0} & =B>0 .
\end{aligned}
$$

By Lemmas 5 and 6 in [7], we deduce that the solution of (1) is nonnegative.

It remains to prove the boundedness of solutions. Then we consider the following function:

$$
F(t)=r_{1} r_{2} r_{3} S(t)+r_{2} r_{3} N(t)+r_{1} r_{3} T(t)+r_{1} V(t) .
$$

Hence, 


$$
\begin{aligned}
D^{\alpha} F(t)= & r_{1} r_{2} r_{3} A+r_{1} B-r_{1} r_{2} r_{3} d S(t)-r_{2} r_{3}\left(d+\varepsilon_{1}\right) N(t) \\
& -r_{1} r_{3}\left(d+\varepsilon_{2}\right) T(t)-r_{1}\left(d+\varepsilon_{3}\right) V(t) \\
\leq & r_{1}\left(r_{2} r_{3} A+B\right)-d F(t) .
\end{aligned}
$$

Then

$$
F(t) \leq F(0) E_{\alpha}\left(-\mathrm{d} t^{\alpha}\right)+\frac{r_{1}}{d}\left(r_{2} r_{3} A+B\right)\left(1-E_{\alpha}\left(-\mathrm{d} t^{\alpha}\right)\right) .
$$

Since $0 \leq E_{\alpha}\left(-\mathrm{d} t^{\alpha}\right) \leq 1$, we have

$$
F(t) \leq F(0)+\frac{r_{1}}{d}\left(r_{2} r_{3} A+B\right),
$$

which implies that $S, N, T$, and $\mathrm{V}$ are bounded. This completes the proof.

Now, we establish the equilibrium points of our model. It is obvious that any equilibrium point of system (1) satisfies the following algebraic equations:

$$
\begin{array}{r}
A-\mathrm{d} S-\beta_{1} S N-\beta_{2} S T=0, \\
r_{1} \beta_{1} S N-\left(d+\varepsilon_{1}\right) N=0, \\
r_{2} \beta_{2} S T-\left(d+\varepsilon_{2}\right) T-\beta_{3} T V=0, \\
B+r_{3} \beta_{3} T V-\left(d+\varepsilon_{3}\right) V=0 .
\end{array}
$$

From (13), we have $N=0$ or $S=\left(d+\varepsilon_{1}\right) / r_{1} \beta_{1}$. Similarly, equation (14) leads to $T=0$ or $r_{2} \beta_{2} S=d+\varepsilon_{2}+\beta_{3} V$ :

(i) For $N=0$ and $T=0$, we have $S=(A / d)$ and $V=B /\left(d+\varepsilon_{3}\right)$. Then system (1) has an equilibrium point of the form $E_{0}\left(S_{0}, 0,0, V_{0}\right)$, where $S_{0}=(A / d)$ and $V_{0}=B /\left(\left(d+\varepsilon_{3}\right)\right)$.

(ii) For $N \neq 0$ and $T=0$, we have $S=\left(d+\varepsilon_{1}\right) / r_{1} \beta_{1}, V=$ $B /\left(d+\varepsilon_{3}\right)$ and $N=\left(d / \beta_{1}\right)\left(\mathscr{A}_{1}-1\right)$, where

$$
\mathscr{A}_{1}=\frac{A r_{1} \beta_{1}}{d\left(d+\varepsilon_{1}\right)} \text {. }
$$

This number reflects the ability of absorbing nutrients by normal cells. It is called absorbing number [4]. When $\mathscr{A}_{1}>1$, system (1) has another equilibrium $E_{1}\left(S_{1}, N_{1}, 0, V_{1}\right)$, where $S_{1}=\left(d+\varepsilon_{1}\right) / r_{1} \beta_{1}$, $N_{1}=\left(d / \beta_{1}\right)\left(\mathscr{A}_{1}-1\right)$, and $V_{1}=B /\left(d+\varepsilon_{3}\right)$. (iii) For $N=0$ and $T \neq 0$, we have $S=\left(\beta_{3} V+d+\right.$ $\left.\varepsilon_{2}\right) / r_{2} \beta_{2}, T=\left(-d / \beta_{2}\right)+\left(A r_{2} / \beta_{3} V+d+\varepsilon_{2}\right)$, and

$$
a_{1} V^{2}+a_{2} V+a_{3}=0 \text {, }
$$

where

$$
\begin{aligned}
& a_{1}=\beta_{3}\left[r_{3} \beta_{3} d+\beta_{2}\left(d+\varepsilon_{3}\right)\right], \\
& a_{2}=\frac{a_{1}}{\beta_{3}}\left(d+\varepsilon_{2}\right)-\beta_{2} \beta_{3}\left(B+r_{2} r_{3} A\right), \\
& a_{3}=-B \beta_{2}\left(d+\varepsilon_{2}\right) .
\end{aligned}
$$

Since $a_{1}>0$ and $a_{3}<0$, we have $\delta=a_{2}^{2}-4 a_{1} a_{3} \geq 0$. Thus equation (17) has two roots given by

$$
V \pm=\frac{-a_{2} \pm \sqrt{\delta}}{2 a_{1}}
$$

Clearly, $V_{+}>0$ and $V_{-}<0$. As $V>0$, we have $V=V_{+}$.

It is obvious that $S>0$. However, $T>0$ implies that $\mathscr{A}_{2}>1+\left(B \beta_{3} /\left(d+\varepsilon_{2}\right)\left(d+\varepsilon_{3}\right)\right)$, where

$$
\mathscr{A}_{2}=\frac{A r_{2} \beta_{2}}{d\left(d+\varepsilon_{2}\right)} .
$$

This number reflects the ability of absorbing nutrients by tumor cells. It can be called the absorbing number of nutrients by tumor cells. Hence, system (1) has another equilibrium point when $\mathscr{A}_{2}>1+\left(B \beta_{3} /\left(d+\varepsilon_{2}\right)\left(d+\varepsilon_{3}\right)\right)$. This equilibrium point is denoted by $E_{2}\left(S_{2}, 0, T_{2}, V_{2}\right)$, where

$$
\begin{aligned}
& V_{2}=V_{+}, \\
& S_{2}=\frac{\beta_{3} V_{2}+d+\varepsilon_{2}}{r_{2} \beta_{2}}, \\
& T_{2}=\frac{-d}{\beta_{2}}+\frac{A r_{2}}{\beta_{3} V_{2}+d+\varepsilon_{2}} .
\end{aligned}
$$

(iv) For $N \neq 0$ and $T \neq 0$, we have $S=\left(d+\varepsilon_{1}\right) / r_{1} \beta_{1}$ and $V=\left(d+\varepsilon_{2} / \beta_{3}\right)\left(\left(\mathscr{A}_{2} / \mathscr{A}_{1}\right)-1\right)$ as $V>0$ implies that $\mathscr{A}_{2}>\mathscr{A}_{1}$. From (15), we get $T=(-B+$ $\left.\left(d+\varepsilon_{3}\right) V\right) / r_{3} \beta_{3} V$. Similarly, $T>0$ leads to $\mathscr{A}_{2}>\mathscr{A}_{1}+\left(A B r_{1} \beta_{1} \beta_{3} / d\left(d+\varepsilon_{3}\right)\left(d+\varepsilon_{2}\right)\left(d+\varepsilon_{1}\right)\right)$.

Substituting $S$ and $T$ in (12), we obtain 


$$
\begin{aligned}
& N=\frac{\left(d+\varepsilon_{2}\right)\left(\left(\mathscr{A}_{2} / \mathscr{A}_{1}\right)-1\right)\left[A r_{1} r_{3} \beta_{1} \beta_{3}-r_{3} \beta_{3} d\left(d+\varepsilon_{1}\right)-\beta_{2}\left(d+\varepsilon_{1}\right)\left(d+\varepsilon_{3}\right)\right]}{r_{3} \beta_{1} \beta_{3}\left(d+\varepsilon_{1}\right)\left(d+\varepsilon_{2}\right)\left(\left(\mathscr{A}_{2} / \mathscr{A}_{1}\right)-1\right)} \\
& +\frac{B \beta_{2} \beta_{3}}{r_{3} \beta_{1} \beta_{3}\left(d+\varepsilon_{2}\right)\left(\left(\mathscr{A}_{2} / \mathscr{A}_{1}\right)-1\right)} . \\
& \mathscr{A}_{1}+\frac{\beta_{2} B}{r_{3} d\left(d+\varepsilon_{2}\right)\left(\left(\mathscr{A}_{2} / \mathscr{A}_{1}\right)-1\right)}>1+\frac{\beta_{2}\left(d+\varepsilon_{3}\right)}{r_{3} \beta_{3} d} \text {. } \\
& \mathscr{A}_{1}+\frac{\beta_{2} B}{r_{3} d\left(d+\varepsilon_{2}\right)\left(\left(A_{2} / A_{1}\right)-1\right)}>1+\frac{\beta_{2}\left(d+\varepsilon_{3}\right)}{r_{3} \beta_{3} d}, \\
& \mathscr{A}_{2}>\mathscr{A}_{1}+\frac{A B r_{1} \beta_{1} \beta_{3}}{d\left(d+\varepsilon_{3}\right)\left(d+\varepsilon_{2}\right)\left(d+\varepsilon_{1}\right)} .
\end{aligned}
$$

Thus, system (1) has another equilibrium point when $\mathscr{A}_{2}>\mathscr{A}_{1}$ : where

This equilibrium point is denoted by $E_{3}\left(S_{3}, N_{3}, T_{3}, V_{3}\right)$,

$$
\begin{aligned}
S_{3}= & \frac{d+\varepsilon_{1}}{r_{1} \beta_{1}}, \\
V_{3}= & \frac{d+\varepsilon_{2}}{\beta_{3}}\left(\left(\mathscr{A}_{2} / \mathscr{A}_{1}\right)-1\right), \\
N_{3}= & \frac{\left(d+\varepsilon_{2}\right)\left(\left(\mathscr{A}_{2} / \mathscr{A}_{1}\right)-1\right)\left[A r_{1} r_{3} \beta_{1} \beta_{3}-r_{3} \beta_{3} d\left(d+\varepsilon_{1}\right)-\beta_{2}\left(d+\varepsilon_{1}\right)\left(d+\varepsilon_{3}\right)\right]}{r_{3} \beta_{1} \beta_{3}\left(d+\varepsilon_{1}\right)\left(d+\varepsilon_{2}\right)\left(\left(\mathscr{A}_{2} / \mathscr{A}_{1}\right)-1\right)} \\
& +\frac{B \beta_{2} \beta_{3}}{r_{3} \beta_{1} \beta_{3}\left(d+\varepsilon_{2}\right)\left(\left(\mathscr{A}_{2} / \mathscr{A}_{1}\right)-1\right)}, \\
T_{3}= & \frac{-B+\left(d+\varepsilon_{3}\right) V_{3}}{r_{3} \beta_{3} V_{3}} .
\end{aligned}
$$

All the above cases are summerized in the following result.

Theorem 2. Let $\mathscr{A}_{1}$ and $\mathscr{A}_{2}$ be defined by (16) and (20). Then

(i) System (1) always has a competition-free equilibrium $E_{0}\left(S_{0}, 0,0, V_{0}\right)$.

(ii) System (1) has a tumor-free equilibrium $E_{1}\left(S_{1}, N_{1}, 0, V_{1}\right)$ when $\mathscr{A}_{1}>1$.

(iii) System (1) has a treatment failure equilibrium $E_{2}\left(S_{2}, 0, T_{2}, V_{2}\right)$ $\mathscr{A}_{2}>1+\left(B \beta_{3} /\left(d+\varepsilon_{2}\right)\left(d+\varepsilon_{3}\right)\right)$.

(iv) System (1) has a partial success equilibrium $E_{3}\left(S_{3}, N_{3}, T_{3}, V_{3}\right)$ when

$$
\begin{aligned}
& \mathscr{A}_{2}>\mathscr{A}_{1}+\frac{A B r_{1} \beta_{1} \beta_{3}}{d\left(d+\varepsilon_{3}\right)\left(d+\varepsilon_{2}\right)\left(d+\varepsilon_{1}\right)}, \\
& \mathscr{A}_{1}+\frac{\beta_{2} B}{r_{3} d\left(d+\varepsilon_{2}\right)\left(\left(\mathscr{A}_{2} / \mathscr{A}_{1}\right)-1\right)}>1+\frac{\beta_{2}\left(d+\varepsilon_{3}\right)}{r_{3} \beta_{3} d} .
\end{aligned}
$$

\section{Stability Analysis}

In this section, we focus on the stability analysis of the equilibria $E_{0}, E_{1}, E_{2}$, and $E_{3}$.

Theorem 3. The competition-free equilibrium $E_{0}$ is globally asymptotically stable for $\mathscr{A}_{2} \leq 1+\left(B \beta_{3} /\left(d+\varepsilon_{2}\right)\left(d+\varepsilon_{3}\right)\right)$ and $\mathscr{A}_{1} \leq 1$, and it is unstable if $\mathscr{A}_{2}>1+\left(B \beta_{3} /\left(d+\varepsilon_{2}\right)(d+\right.$ $\left.\varepsilon_{3}\right)$ ) or $\mathscr{A}_{1}>1$.

Proof. In order to show the first part of this theorem, we consider the following Lyapunov functional:

$$
L_{0}(t)=S_{0} \phi\left(\frac{S(t)}{S_{0}}\right)+\frac{1}{r_{1}} N(t)+\frac{1}{r_{2}} T(t)+\frac{1}{r_{2} r_{3}} V_{0} \phi\left(\frac{V(t)}{V_{0}}\right) \text {, }
$$

where $\phi(x)=x-\ln (x)-1$ for $x>0$.

Based on the property of fractional derivatives given in [8], we get 


$$
\begin{aligned}
D^{\alpha} L_{0} \leq & \left(1-\frac{S_{0}}{S}\right) D^{\alpha} S+\frac{1}{r_{1}} D^{\alpha} N+\frac{1}{r_{2}} D^{\alpha} T+\frac{1}{r_{2} r_{3}}\left(1-\frac{V_{0}}{V}\right) D^{\alpha} V \\
= & \left(1-\frac{S_{0}}{S}\right)\left(A-\mathrm{d} S-\beta_{1} S N-\beta_{2} S T\right) \\
& +\frac{1}{r_{1}}\left(r_{1} \beta_{1} S N-\left(d+\varepsilon_{1}\right) N\right) \\
& +\frac{1}{r_{2}}\left(r_{2} \beta_{2} S T-\left(d+\varepsilon_{2}\right) T-\beta_{3} T V\right) \\
& +\frac{1}{r_{2} r_{3}}\left(1-\frac{V_{0}}{V}\right)\left(B+r_{3} \beta_{3} T V-\left(d+\varepsilon_{3}\right) V\right) .
\end{aligned}
$$

By $S_{0}=(A / d)$ and $V_{0}=B /\left(d+\varepsilon_{3}\right)$, we obtain

$$
\begin{aligned}
D^{\alpha} L_{0} \leq & \mathrm{d} S_{0}\left(1-\frac{S}{S_{0}}\right)\left(1-\frac{S_{0}}{S}\right)+\beta_{1} S_{0} N+\beta_{2} S_{0} T-\frac{d+\varepsilon_{1}}{r_{1}} N-\frac{d+\varepsilon_{2}}{r_{2}} T \\
& +\frac{d+\varepsilon_{3}}{r_{2} r_{3}} V_{0}\left(1-\frac{V}{V_{0}}\right)\left(1-\frac{V_{0}}{V}\right)-\frac{\beta_{3}}{r_{2}} T V_{0} \\
= & \frac{-d}{S}\left(S-S_{0}\right)^{2}+\left(\beta_{1} S_{0}-\frac{d+\varepsilon_{1}}{r_{1}}\right) N+\left(\beta_{2} S_{0}-\frac{d+\varepsilon_{2}}{r_{2}}-\frac{\beta_{3} V_{0}}{r_{2}}\right) T \\
& -\frac{d+\varepsilon_{3}}{r_{2} r_{3}} \frac{\left(V-V_{0}\right)^{2}}{V} \\
= & \frac{d}{S}\left(S-S_{0}\right)^{2}+\frac{d+\varepsilon_{1}}{r_{1}}\left(\mathscr{A}_{1}-1\right) N \\
& +\frac{d+\varepsilon_{2}}{r_{2}}\left(\mathscr{A}_{2}-1-\frac{B \beta_{3}}{\left(d+\varepsilon_{2}\right)\left(d+\varepsilon_{3}\right)}\right) T-\frac{d+\varepsilon_{3}}{r_{2} r_{3}} \frac{\left(V-V_{0}\right)^{2}}{V} .
\end{aligned}
$$

Then $D^{\alpha} L_{0} \leq 0 \quad$ when $\quad \mathscr{A}_{1} \leq 1 \quad$ and $\quad \mathscr{A}_{2} \leq 1+$ $\left(B \beta_{3} /\left(d+\varepsilon_{2}\right)\left(d+\varepsilon_{3}\right)\right)$. Clearly, $D^{\alpha} L_{0}=0$ if and only if $S=S_{0}, N=0, T=0$, and $V=V_{0}$. Then the largest invariant set contained in $\left\{(S, N, T, V) \mid D^{\alpha} L_{0}(t)=0\right\}$ is the singleton $\left\{E_{0}\right\}$. By LaSalle's invariance principale [9], we deduce that $E_{0}$ is globally asymptotically stable for $\mathscr{A}_{1} \leq 1$ and $\mathscr{A}_{2} \leq 1+\left(B \beta_{3} /\left(d+\varepsilon_{2}\right)\left(d+\varepsilon_{3}\right)\right)$.

It remains to investigate the dynamical property of $E_{0}$ in case when $\mathscr{A}_{1}>1$ or $\mathscr{A}_{2}>1+\left(B \beta_{3} /\left(d+\varepsilon_{2}\right)\left(d+\varepsilon_{3}\right)\right)$. For this purpose, we compute the characteristic equation at $E_{0}$ that is given by

$$
\left(\lambda-\lambda_{1}\right)\left(\lambda-\lambda_{2}\right)\left(\lambda-\lambda_{3}\right)\left(\lambda-\lambda_{4}\right)=0,
$$

where

$$
\begin{aligned}
& \lambda_{1}=-d, \\
& \lambda_{2}=-d-\varepsilon_{3}, \\
& \lambda_{3}=\left(d+\varepsilon_{2}\right)\left(\mathscr{A}_{2}-1-\frac{B \beta_{3}}{\left(d+\varepsilon_{2}\right)\left(d+\varepsilon_{3}\right)}\right), \\
& \lambda_{4}=\left(d+\varepsilon_{1}\right)\left(\mathscr{A}_{1}-1\right) .
\end{aligned}
$$

We have $\lambda_{1}<0, \quad \lambda_{2}<0, \quad \lambda_{3}>0$ if $\mathscr{A}_{2}>1+\left(B \beta_{3}\right)$ $\left.\left(d+\varepsilon_{2}\right)\left(d+\varepsilon_{3}\right)\right)$, and $\lambda_{4}>0$ if $\mathscr{A}_{1}>1$. Consequently, $E_{0}$ is unstable if $\mathscr{A}_{1}>1$ or $\mathscr{A}_{2}>1+\left(B \beta_{3} /\left(d+\varepsilon_{2}\right)\left(d+\varepsilon_{3}\right)\right)$. 
Theorem 4. Suppose that $\mathscr{A}_{1}>1$. Then the tumor-free equilibrium $E_{1}$ is globally asymptotically stable if

$$
\mathscr{A}_{2} \leq \mathscr{A}_{1}+\frac{A B r_{1} \beta_{1} \beta_{3}}{d\left(d+\varepsilon_{1}\right)\left(d+\varepsilon_{2}\right)\left(d+\varepsilon_{3}\right)},
$$

and it is unstable if

$$
\mathscr{A}_{2}>\mathscr{A}_{1}+\frac{A B r_{1} \beta_{1} \beta_{3}}{d\left(d+\varepsilon_{1}\right)\left(d+\varepsilon_{2}\right)\left(d+\varepsilon_{3}\right)} .
$$

Proof. Consider the following Lyapunov functional:

$$
L_{1}(t)=S_{1} \phi\left(\frac{S(t)}{S_{1}}\right)+\frac{1}{r_{1}} N_{1} \phi\left(\frac{N(t)}{N_{1}}\right)+\frac{1}{r_{2}} T(t)+\frac{1}{r_{2} r_{3}} V_{1} \phi\left(\frac{V(t)}{V_{1}}\right) .
$$

Then

$$
\begin{aligned}
D^{\alpha} L_{1} \leq & \left(1-\frac{S_{1}}{S}\right)\left(A-\mathrm{d} S-\beta_{1} S N-\beta_{2} S T\right) \\
& +\frac{1}{r_{1}}\left(1-\frac{N_{1}}{N}\right)\left(r_{1} \beta_{1} S N-\left(d+\varepsilon_{1}\right) N\right) \\
& +\frac{1}{r_{2}}\left(r_{2} \beta_{2} S T-\left(d+\varepsilon_{2}\right) T-\beta_{3} T V\right) \\
& +\frac{1}{r_{2} r_{3}}\left(1-\frac{V_{1}}{V}\right)\left(B+r_{3} \beta_{3} T V-\left(d+\varepsilon_{3}\right) V\right) .
\end{aligned}
$$

By $V_{1}=B /\left(d+\varepsilon_{3}\right)$ and $S_{1}=\left(d+\varepsilon_{1}\right) / r_{1} \beta_{1}$, we obtain

$$
\begin{aligned}
& D^{\alpha} L_{1} \leq \mathrm{d} S_{1}\left(1-\frac{S_{1}}{S}\right)\left(1-\frac{S}{S_{1}}\right)+\beta_{1} S_{1} N_{1}\left(2-\frac{S_{1}}{S}-\frac{S}{S_{1}}\right) \\
&+\left(\beta_{1} S_{1}-\frac{d+\varepsilon_{1}}{r_{1}}\right) N+\left(\beta_{2} \frac{d+\varepsilon_{1}}{r_{1} \beta_{1}}-\frac{d+\varepsilon_{2}}{r_{2}}-\frac{\beta_{3} B}{r_{2}\left(d+\varepsilon_{3}\right)}\right) T \\
&+\frac{B}{r_{2} r_{3}}\left(2-\frac{V_{1}}{V}-\frac{V}{V_{1}}\right) \\
&=-\left(d+\beta_{1} N_{1}\right) \frac{\left(S-S_{1}\right)^{2}}{S}-\frac{B}{r_{2} r_{3}} \frac{\left(V-V_{1}\right)^{2}}{V V_{1}} \\
&+\frac{d\left(d+\varepsilon_{1}\right)\left(d+\varepsilon_{2}\right)}{A r_{1} r_{2} \beta_{1}}\left(\mathscr{A}_{2}-\mathscr{A}_{1}-\frac{A B r_{1} \beta_{1} \beta_{3}}{d\left(d+\varepsilon_{1}\right)\left(d+\varepsilon_{2}\right)\left(d+\varepsilon_{3}\right)}\right) T . \\
& \mathscr{A}_{2} \leq \mathscr{A}_{1}+\frac{A B r_{1} \beta_{1} \beta_{3}}{d\left(d+\varepsilon_{1}\right)\left(d+\varepsilon_{2}\right)\left(d+\varepsilon_{3}\right)}
\end{aligned}
$$

Then $D^{\alpha} L_{1} \leq 0$ when

$$
\mathscr{A}_{2} \leq \mathscr{A}_{1}+\frac{A B r_{1} \beta_{1} \beta_{3}}{d\left(d+\varepsilon_{1}\right)\left(d+\varepsilon_{2}\right)\left(d+\varepsilon_{3}\right)} .
$$

Obviously, $D^{\alpha} L_{1}=0$ if and only if $S=S_{1}, N=N_{1}, T=0$, and $V=V_{1}$. Then the largest invariant set contained in $\left\{(S, N, T, V) \mid D^{\alpha} L_{1}(t)=0\right\}$ is the singleton $E_{1}$. By LaSalle's invariance principle, we deduce that $E_{1}$ is globally asymptotically stable for by

On the contrary, the characteristic equation at $E_{1}$ is given

$$
\left(d+\varepsilon_{1}+\lambda\right)\left(r_{2} \beta_{2} S_{1}-d-\varepsilon_{2}-\beta_{3} V_{1}-\lambda\right) f(\lambda)=0,
$$

where

$f(\lambda)=\left(d+\lambda+\beta_{1} N_{1}\right)\left(d+\varepsilon_{1}+\lambda-r_{1} \beta_{1} S_{1}\right)+r_{1} \beta_{1}^{2} N_{1} S_{1}$.

One of the eigenvalues of (39) is 


$$
\begin{aligned}
\lambda_{1} & =r_{2} \beta_{2} S_{1}-d-\varepsilon_{2}-\beta_{3} V_{1} \\
& =\frac{d\left(d+\varepsilon_{1}\right)\left(d+\varepsilon_{2}\right)}{A r_{1} \beta_{1}}\left(\mathscr{A}_{2}-\mathscr{A}_{1}-\frac{A B r_{1} \beta_{1} \beta_{3}}{d\left(d+\varepsilon_{1}\right)\left(d+\varepsilon_{2}\right)\left(d+\varepsilon_{3}\right)}\right) .
\end{aligned}
$$

We observe that $\lambda_{1}>0$ if

$$
\mathscr{A}_{2}>\mathscr{A}_{1}+\frac{A B r_{1} \beta_{1} \beta_{3}}{d\left(d+\varepsilon_{1}\right)\left(d+\varepsilon_{2}\right)\left(d+\varepsilon_{3}\right)} .
$$

Thus, $E_{1}$ is unstable when

$$
\mathscr{A}_{2}>\mathscr{A}_{1}+\frac{A B r_{1} \beta_{1} \beta_{3}}{d\left(d+\varepsilon_{1}\right)\left(d+\varepsilon_{2}\right)\left(d+\varepsilon_{3}\right)} .
$$

Theorem 5. Suppose that $\mathscr{A}_{2}>1+\left(B \beta_{3} /\left(d+\varepsilon_{2}\right)\left(d+\varepsilon_{3}\right)\right)$ and $\left(\mathscr{A}_{2} / \mathscr{A}_{1}\right)>1$. Then the treatment failure equilibrium $E_{2}$ is globally asymptotically stable if

$$
1+\frac{\beta_{2}\left(d+\varepsilon_{3}\right)}{r_{3} \beta_{3} d} \geq \mathscr{A}_{1}+\frac{B \beta_{2}}{r_{3} d\left(d+\varepsilon_{2}\right)\left(\left(\mathscr{A}_{2} / \mathscr{A}_{1}\right)-1\right)},
$$

and becomes unstable if

$$
1+\frac{\beta_{2}\left(d+\varepsilon_{3}\right)}{r_{3} \beta_{3} d}<\mathscr{A}_{1}+\frac{B \beta_{2}}{r_{3} d\left(d+\varepsilon_{2}\right)\left(\left(\mathscr{A}_{2} / \mathscr{A}_{1}\right)-1\right)} .
$$

Proof. Consider the following Lyapunov functional:

$$
\begin{aligned}
L_{2}(t)= & S_{2} \phi\left(\frac{S(t)}{S_{2}}\right)+\frac{1}{r_{1}} N(t)+\frac{1}{r_{2}} T_{2} \phi\left(\frac{T(t)}{T_{2}}\right) \\
& +\frac{1}{r_{2} r_{3}} V_{2} \phi\left(\frac{V(t)}{V_{2}}\right) .
\end{aligned}
$$

Then

$$
\begin{aligned}
D^{\alpha} L_{2} \leq & \mathrm{d} S_{2}\left(1-\frac{S_{2}}{S}\right)\left(1-\frac{S}{S_{2}}\right)+\beta_{2} S_{2} T_{2}\left(2-\frac{S_{2}}{S}-\frac{S}{S_{2}}\right) \\
& +\left(\beta_{1} S_{2}-\frac{d+\varepsilon_{1}}{r_{1}}\right) N \\
& +\left(\beta_{2} S_{2}-\frac{d+\varepsilon_{2}}{r_{2}}-\frac{\beta_{3}}{r_{2}} V_{2}\right) T \\
& +\frac{B}{r_{2} r_{3}}\left(2-\frac{V_{2}}{V}-\frac{V}{V_{2}}\right) \\
= & -\left(d+\beta_{2} T_{2}\right) \frac{\left(S-S_{2}\right)^{2}}{S}+\beta_{1}\left(S_{2}-S_{3}\right) N-\frac{B}{r_{2} r_{3}} \frac{\left(V-V_{2}\right)^{2}}{V V_{2}} .
\end{aligned}
$$

By computation, we find

$$
\begin{aligned}
S_{2}-S_{3}= & A r_{1} \beta_{1} r_{3} \beta_{3}\left(d+\varepsilon_{2}\right)\left(\frac{A_{2}}{A_{1}}-1\right)+B \beta_{2} \beta_{3}\left(d+\varepsilon_{1}\right) \\
& -\beta_{2}\left(d+\varepsilon_{1}\right)\left(d+\varepsilon_{2}\right)\left(d+\varepsilon_{3}\right)\left(\frac{A_{2}}{A_{1}}-1\right) \\
& -r_{3} \beta_{3} d\left(d+\varepsilon_{1}\right)\left(d+\varepsilon_{2}\right)\left(\frac{A_{2}}{A_{1}}-1\right) .
\end{aligned}
$$

Thus, $S_{2}-S_{3} \leq 0$ implies that

$$
\mathscr{A}_{1}+\frac{B \beta_{2}}{r_{3} d\left(d+\varepsilon_{2}\right)\left(\left(\mathscr{A}_{2} / \mathscr{A}_{1}\right)-1\right)} \leq 1+\frac{\beta_{2}\left(d+\varepsilon_{3}\right)}{r_{3} \beta_{3} d} \text {. }
$$

Consequently, $D^{\alpha} L_{2} \leq 0$ when

$$
\mathscr{A}_{1}+\frac{B \beta_{2}}{r_{3} d\left(d+\varepsilon_{2}\right)\left(\left(\mathscr{A}_{2} / \mathscr{A}_{1}\right)-1\right)} \leq 1+\frac{\beta_{2}\left(d+\varepsilon_{3}\right)}{r_{3} \beta_{3} d} \text {. }
$$

Clearly, $D^{\alpha} L_{2}=0$ if and only if $S=S_{2}, N=0, T=T_{2}$, and $V=V_{2}$. Then the largest invariant set contained in $\left\{(S, N, T, V) \mid D^{\alpha} L_{2}(t)=0\right\}$ is the singleton $\left\{E_{2}\right\}$. By LaSalle's invariance principle, we deduce that $E_{2}$ is globally asymptotically stable for

$$
1+\frac{\beta_{2}\left(d+\varepsilon_{3}\right)}{r_{3} \beta_{3} d} \geq \mathscr{A}_{1}+\frac{B \beta_{2}}{r_{3} d\left(d+\varepsilon_{2}\right)\left(\left(\mathscr{A}_{2} / \mathscr{A}_{1}\right)-1\right)} .
$$

On the other side, the characteristic equation at $E_{2}$ is given by

$$
\left(r_{1} \beta_{1} S_{2}-d-\varepsilon_{1}-\lambda\right) g(\lambda)=0
$$

where 


$$
\begin{aligned}
g(\lambda)= & \left(d+\beta_{2} T_{2}+\lambda\right)\left[\left(d+\varepsilon_{2}+\lambda+\beta_{3} V_{2}-r_{2} \beta_{2} S_{2}\right)\left(r_{3} \beta_{3} T_{2}-d-\varepsilon_{3}-\lambda\right)-r_{3} \beta_{3}^{2} T_{2} V_{2}\right] \\
& +r_{2} \beta_{2}^{2} S_{2} T_{2}\left(r_{3} \beta_{3} T_{2}-d-\varepsilon_{3}-\lambda\right) .
\end{aligned}
$$

One of the eigenvalues of (51) is

$$
\lambda_{2}=r_{1} \beta_{1} S_{2}-d-\varepsilon_{1}=r_{1} \beta_{1}\left(S_{2}-S_{3}\right) .
$$

We can observe from the proof of part (a) that $\lambda_{2}>0$ if

$1+\frac{\beta_{2}\left(d+\varepsilon_{3}\right)}{r_{3} \beta_{3} d}<\mathscr{A}_{1}+\frac{B \beta_{2}}{r_{3} d\left(d+\varepsilon_{2}\right)\left(\left(\mathscr{A}_{2} / \mathscr{A}_{1}\right)-1\right)}$.

Thus, $E_{2}$ is unstable when

$$
1+\frac{\beta_{2}\left(d+\varepsilon_{3}\right)}{r_{3} \beta_{3} d}<\mathscr{A}_{1}+\frac{B \beta_{2}}{r_{3} d\left(d+\varepsilon_{2}\right)\left(\left(\mathscr{A}_{2} / \mathscr{A}_{1}\right)-1\right)} .
$$

Theorem 6. The partial success equilibrium $E_{3}$ is globally asymptotically stable if

$$
\begin{aligned}
& \mathscr{A}_{2}>\mathscr{A}_{1}+\frac{A B r_{1} \beta_{1} \beta_{3}}{d\left(d+\varepsilon_{1}\right)\left(d+\varepsilon_{2}\right)\left(d+\varepsilon_{3}\right)}, \\
& \mathscr{A}_{1}+\frac{B \beta_{2}}{r_{3} d\left(d+\varepsilon_{2}\right)\left(\left(\mathscr{A}_{2} / \mathscr{A}_{1}\right)-1\right)}>1+\frac{\beta_{2}\left(d+\varepsilon_{3}\right)}{r_{3} \beta_{3} d} .
\end{aligned}
$$

Proof. Consider the following Lyapunov functional

$$
\begin{aligned}
L_{3}(t)= & S_{3} \phi\left(\frac{S(t)}{S_{3}}\right)+\frac{1}{r_{1}} N_{3} \phi\left(\frac{N(t)}{N_{3}}\right)+\frac{1}{r_{2}} T_{3} \phi\left(\frac{T(t)}{T_{3}}\right) \\
& +\frac{1}{r_{2} r_{3}} V_{3} \phi\left(\frac{V(t)}{V_{3}}\right) .
\end{aligned}
$$

Then

$$
\begin{aligned}
D^{\alpha} L_{3} \leq & \left(1-\frac{S_{3}}{S}\right)\left(A-\mathrm{d} S-\beta_{1} S N-\beta_{2} S T\right) \\
& +\frac{1}{r_{1}}\left(1-\frac{N_{3}}{N}\right)\left(r_{1} \beta_{1} S N-\left(d+\varepsilon_{1}\right) N\right) \\
& +\frac{1}{r_{2}}\left(1-\frac{T_{3}}{T}\right)\left(r_{2} \beta_{2} S T-\left(d+\varepsilon_{2}\right) T-\beta_{3} T V\right) \\
& +\frac{1}{r_{2} r_{3}}\left(1-\frac{V_{3}}{V}\right)\left(B+r_{3} \beta_{3} T V-\left(d+\varepsilon_{3}\right) V\right) .
\end{aligned}
$$

By $A=\mathrm{d} S_{3}+\beta_{2} S_{3} T_{3}+\beta_{1} S_{3} N_{3}, \quad\left(\beta_{3} / r_{2}\right) T_{3} V_{3}=\left(d+\varepsilon_{3} /\right.$ $\left.r_{2} r_{3}\right) V_{3}-\left(B / r_{2} r_{3}\right), \beta_{1} S_{3} N_{3}=\left(d+\varepsilon_{1} / r_{1}\right) N_{3}$, and $\beta_{2} S_{3} T_{3}=$ $\left(d+\varepsilon_{2} / r_{2}\right) T_{3}+\left(\beta_{3} / r_{2}\right) T_{3} V_{3}$, we get

$$
\begin{aligned}
D^{\alpha} L_{3} \leq & \mathrm{d} S_{3}\left(1-\frac{S_{3}}{S}\right)\left(1-\frac{S}{S_{3}}\right)+\beta_{2} S_{3} T_{3}\left(2-\frac{S_{3}}{S}-\frac{S}{S_{3}}\right) \\
& +\left(\beta_{1} S_{3}-\frac{d+\varepsilon_{1}}{r_{1}}\right) N+\left(\beta_{2} S_{3}-\frac{d+\varepsilon_{2}}{r_{2}}-\frac{\beta_{3}}{r_{2}} V_{3}\right) T \\
& +\beta_{1} S_{3} N_{3}\left(2-\frac{S_{3}}{S}-\frac{S}{S_{3}}\right)+\frac{B}{r_{2} r_{3}}\left(2-\frac{V_{3}}{V}-\frac{V}{V_{3}}\right) \\
= & -\left(d+\beta_{2} T_{3}+\beta_{1} N_{3}\right) \frac{\left(S-S_{3}\right)^{2}}{S}-\frac{B}{r_{2} r_{3}} \frac{\left(V-V_{3}\right)^{2}}{V V_{3}} .
\end{aligned}
$$

Therefore $D^{\alpha} L_{3} \leq 0$, with equality if and only if $S=S_{3}$ and $V=V_{3}$. By a simple computation, we show that $D^{\alpha} L_{3}=$ 0 if and only if $S=S_{3}, N=N_{3}, T=T_{3}$ and $V=V_{3}$. It follows from LaSalle's invariance principale that $E_{3}$ is globally asymptotically stable under the conditions that this point exists.

\section{Numerical Simulations}

In this section, we give some numerical simulations to illustrate and validate our theoretical results, and we present some biological interpretations. We choose the time interval from $t=0$ to $t=400$ with a step size $\Delta t=0.1$. We take $A=0.02, d=0.02, B=0.01, r_{1}=0.8$, and $r_{3}=0.5$. The parameters $\beta_{1}, \beta_{2}, \beta_{3}, \varepsilon_{1}, \varepsilon_{2}, \varepsilon_{3}$, and $r_{2}$ of model (1) are taken as free parameters.

First, we take $\beta_{1}=0.03, \beta_{2}=0.03, \beta_{3}=0.1, \varepsilon_{1}=0.04$, $\varepsilon_{2}=0.01, \quad \varepsilon_{3}=0.008$, and $r_{2}=0.8$. These values give $\mathscr{A}_{1}=0.4, \quad \mathscr{A}_{2}=0.8$, and $1+\left(B \beta_{3} /\left(d+\varepsilon_{2}\right)\left(d+\varepsilon_{3}\right)\right)=$ 2.1905. Thus, $\mathscr{A}_{1}<1$ and $\mathscr{A}_{2}<1+\left(B \beta_{3} /\left(d+\varepsilon_{2}\right)\left(d+\varepsilon_{3}\right)\right)$. According to Theorem 1, the equilibrium $E_{0}(1,0,0,0.3571)$ is globally asymptotically stable which consists with our numerical simulation in Figure 1. This may reflect an extreme competition between normal and tumor cells, leading to the extinction of normal cells and eradicating tumor cells by the M1 virus, giving rise to patient death.

Next, we take $\beta_{1}=0.1, \beta_{2}=0.03, \beta_{3}=0.1, \varepsilon_{1}=0.008$, $\varepsilon_{2}=0.01, \varepsilon_{3}=0.006$, and $r_{2}=0.8$. For this case, we obtain

$$
\begin{aligned}
& \mathscr{A}_{1}=2.8571, \\
& \mathscr{A}_{2}=0.8, \\
& \mathscr{A}_{1}+\frac{A B r_{1} \beta_{1} \beta_{3}}{d\left(d+\varepsilon_{1}\right)\left(d+\varepsilon_{2}\right)\left(d+\varepsilon_{3}\right)}=6.5201 .
\end{aligned}
$$

Thus we get 


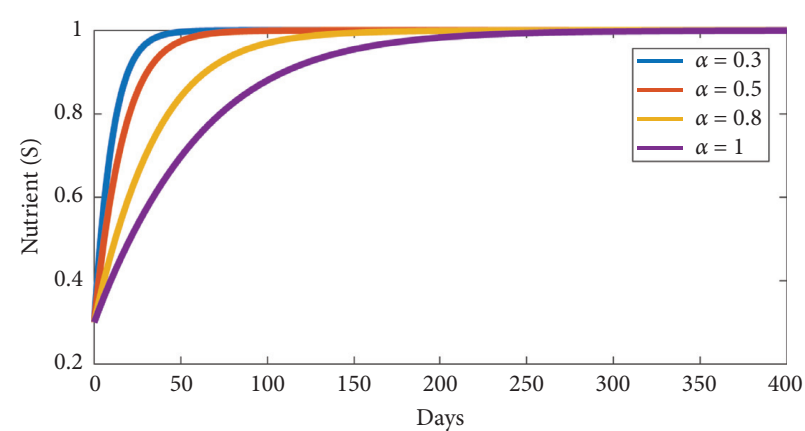

(a)

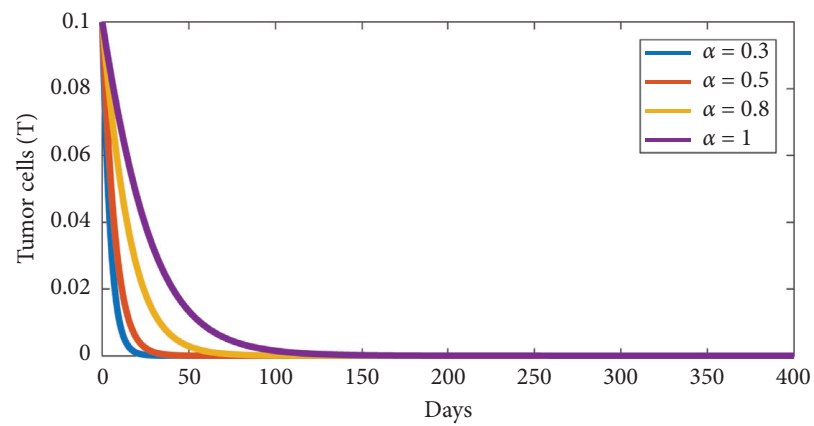

(c)

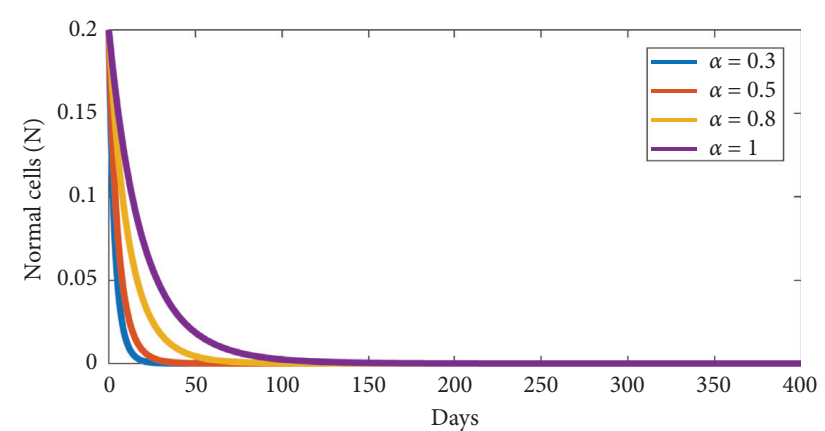

(b)

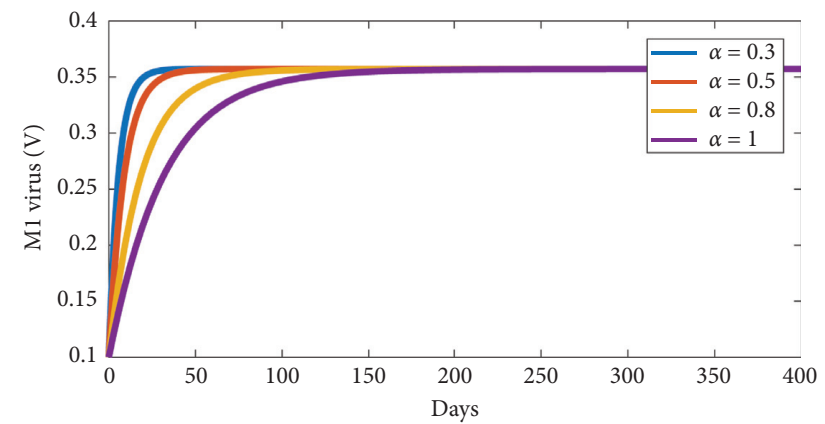

(d)

Figure 1: Stability of the competition-free equilibrium $E_{0}$.

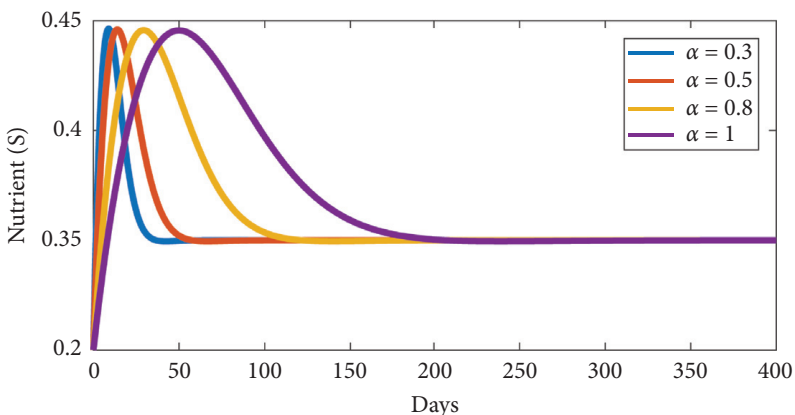

(a)

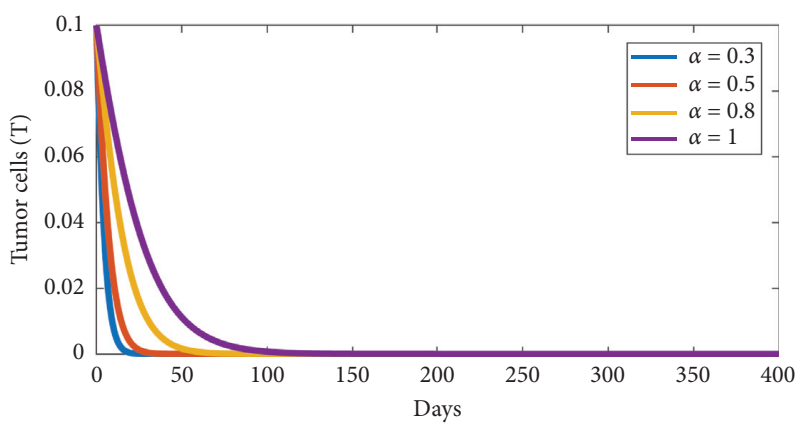

(c)

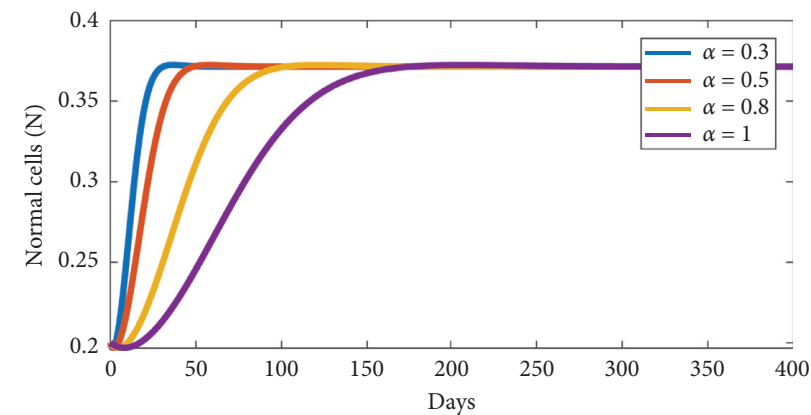

(b)

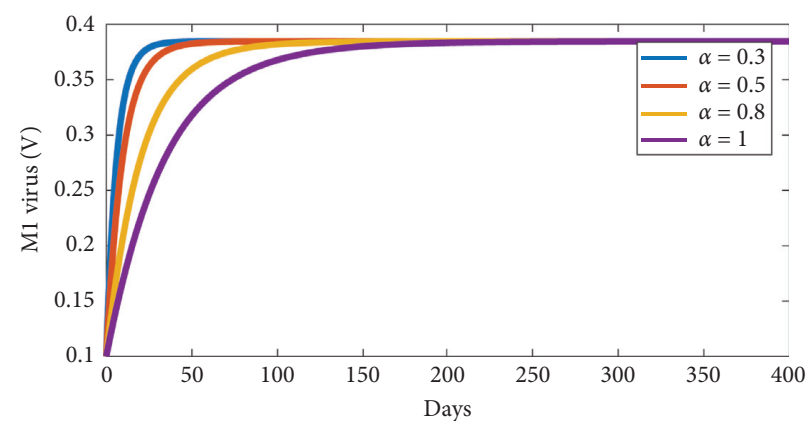

(d)

FIgURe 2: Stability of the tumor-free equilibrium $E_{1}$. 


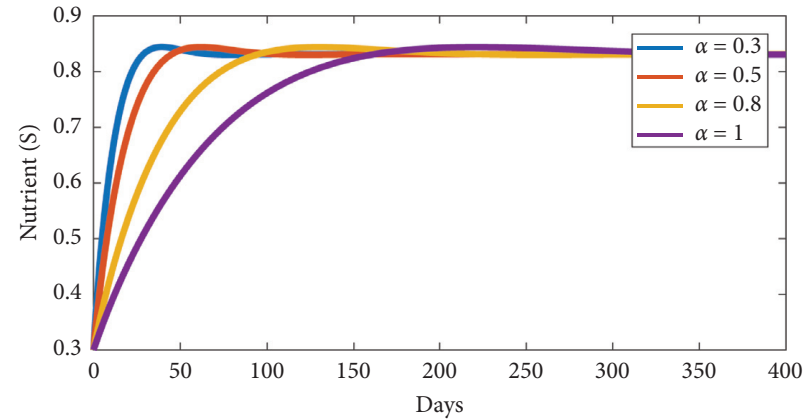

(a)

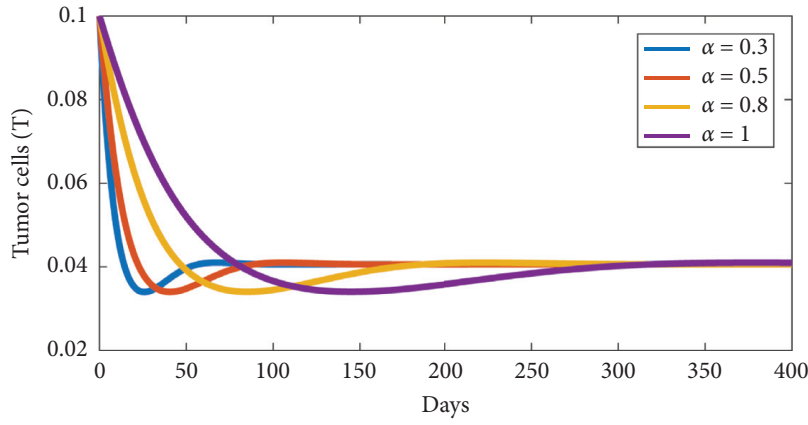

(c)

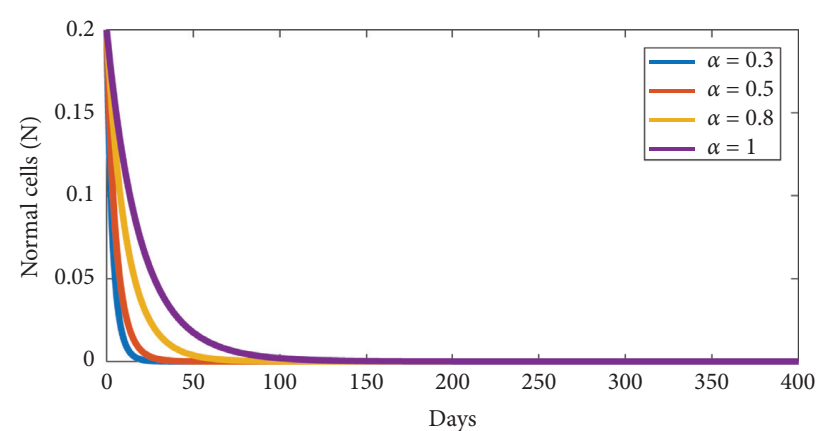

(b)

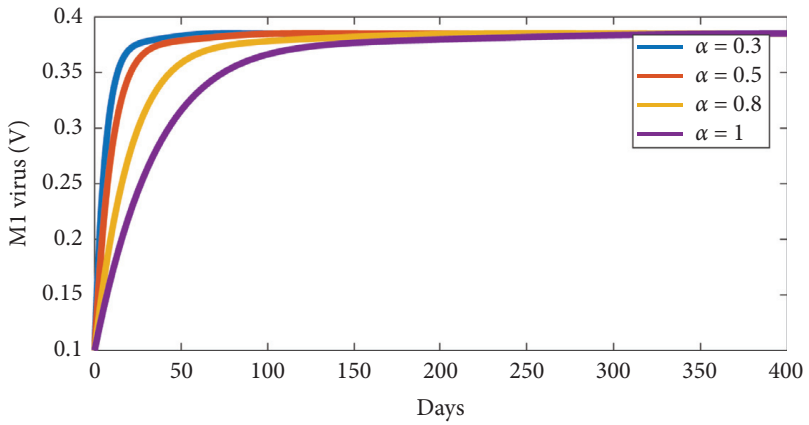

(d)

FIgURE 3: Stability of the treatment failure equilibrium $E_{2}$.

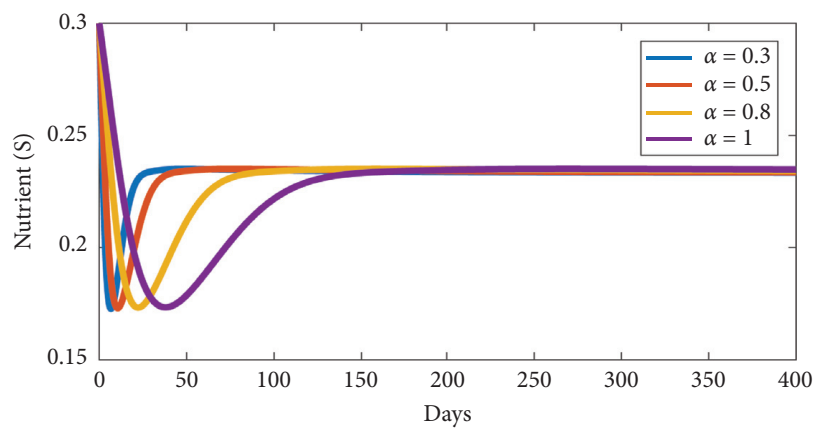

(a)

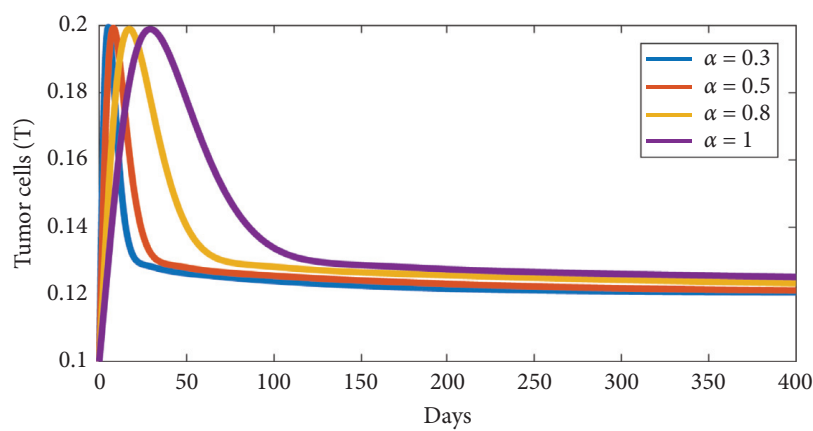

(c)

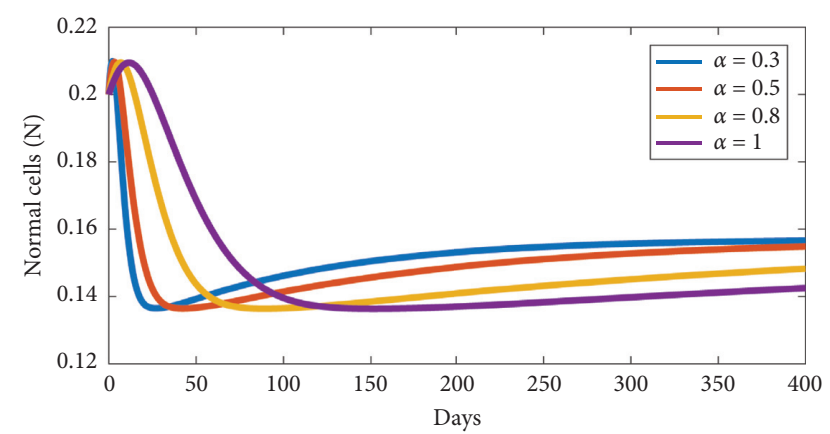

(b)

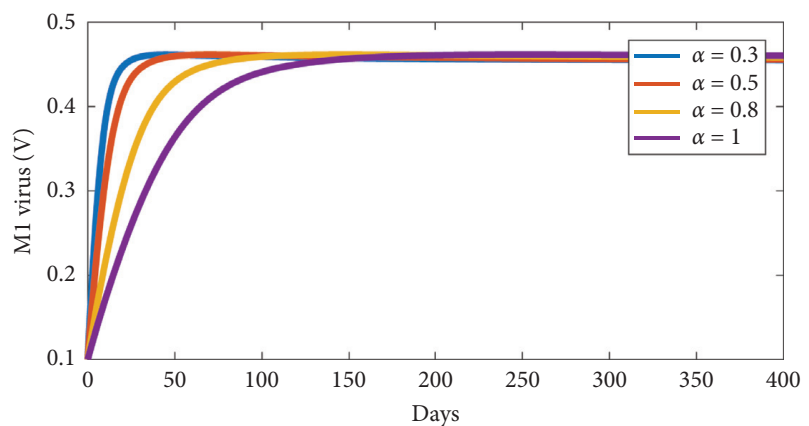

(d)

FIgURE 4: Stability of the partial success equilibrium $E_{3}$. 


$$
\begin{aligned}
& \mathscr{A}_{1}>1, \\
& \mathscr{A}_{2} \leq \mathscr{A}_{1}+\frac{A B r_{1} \beta_{1} \beta_{3}}{d\left(d+\varepsilon_{1}\right)\left(d+\varepsilon_{2}\right)\left(d+\varepsilon_{3}\right)} .
\end{aligned}
$$

In agreement with Theorem 4 , the tumor-free equilibrium $E_{1}(0.35,0.3714,0,0.3846)$ is globally asymptotically stable as exhibited in Figure 2. In this situation, we notice that the M1 virotherapy successfully eliminates tumor cells, and then normal cells have been restored. Consequently, the patient's health will be improved.

In Figure 3, we assume that $\beta_{1}=0.03, \beta_{2}=0.1, \beta_{3}=0.1$, $\varepsilon_{1}=0.04, \varepsilon_{2}=0.008, \varepsilon_{3}=0.008$, and $r_{2}=0.8$. Thus,

$$
\begin{aligned}
\mathscr{A}_{2}= & 2.8571>2.2755=1+\frac{B \beta_{3}}{\left(d+\varepsilon_{2}\right)\left(d+\varepsilon_{3}\right)}, \\
\frac{\mathscr{A}_{2}}{\mathscr{A}_{1}}= & 7.1429>1, \\
1+\frac{\beta_{2}\left(d+\varepsilon_{3}\right)}{r_{3} \beta_{3} d}= & 3.8 \geq 0.9814=\mathscr{A}_{1} \\
& +\frac{B \beta_{2}}{r_{3} d\left(d+\varepsilon_{2}\right)\left(\left(\mathscr{A}_{2} / \mathscr{A}_{1}\right)-1\right)} .
\end{aligned}
$$

We can see that the equilibrium $E_{2}(0.8313$, $0,0.0406,0.385)$ is globally asymptotically stable that agrees with our result in Theorem 5. Biologically, our treatment fails in eliminating the tumor cells as that normal cells are lost. Hence, the patient's health is in danger.

Finally, we choose $\beta_{1}=0.15, \beta_{2}=0.35, \beta_{3}=0.1$, $\varepsilon_{1}=0.008, \varepsilon_{2}=0.008, \varepsilon_{3}=0.008$, and $r_{2}=0.9$. We get

$$
\begin{gathered}
\mathscr{A}_{2}=11.25>4.2857=\mathscr{A}_{1}, \\
\mathscr{A}_{2}>9.7522=\mathscr{A}_{1}+\frac{A B r_{1} \beta_{1} \beta_{3}}{d\left(d+\varepsilon_{1}\right)\left(d+\varepsilon_{2}\right)\left(d+\varepsilon_{3}\right)}, \\
1+\frac{\beta_{2}\left(d+\varepsilon_{3}\right)}{r_{3} \beta_{3} d}=10.8<11.978=\mathscr{A}_{1}+\frac{B \beta_{2}}{r_{3} d\left(d+\varepsilon_{2}\right)\left(\left(\mathscr{A}_{2} / \mathscr{A}_{1}\right)-1\right)} .
\end{gathered}
$$

In agreement with Theorem 6, the equilibrium $E_{3}(0.2333,0.1571,0.1204,0.455)$ is globally asymptotically stable as shown in Figure 4. Here, our treatment partially reduces tumor cells and increases normal cells' levels. However, the treatment cannot wholly eliminate tumor cells, but it can prolong the patient's life.

\section{Conclusion}

In this paper, we have studied the dynamics of an oncolytic M1 virotherapy model, considering the memory effect denoted by the Caputo fractional derivative. The well-posedness of the proposed model was proved through nonnegativity and boundedness of solutions. We found that the model has four possible equilibrium points, namely, the competition-free equilibrium $E_{0}$, the tumor-free equilibrium $E_{1}$, the treatment failure equilibrium $E_{2}$, and the partial success equilibrium $E_{3}$. By constructing suitable Lyapunov functionals, the global stability of $E_{0}$ is determined by two threshold parameters that are the absorbing number of nutrients by normal cells $\mathscr{A}_{1}$ and the absorbing number of nutrients by tumor cells $\mathscr{A}_{2}$, when $\mathscr{A}_{1} \leq 1$ and $\mathscr{A}_{2} \leq 1+\left(B \beta_{3} /\left(d+\varepsilon_{2}\right)\left(d+\varepsilon_{3}\right)\right), E_{0}$ is globally asymptotically stable, and these conditions determine when normal and tumor cells are lost, which may not be useful to test the viability of treatment. The tumor-free equilibrium $E_{1}$ exists and is globally asymptotically stable if $\mathscr{A}_{1}>1$ and

$$
\mathscr{A}_{2} \leq \mathscr{A}_{1}+\frac{A B r_{1} \beta_{1} \beta_{3}}{d\left(d+\varepsilon_{1}\right)\left(d+\varepsilon_{2}\right)\left(d+\varepsilon_{3}\right)},
$$

and these conditions show that the M1 virus succeeds to eliminate the tumor, which is helpful in improving virotherapy. The treatment failure equilibrium $E_{2}$ exists and is globally asymptotically stable if 


$$
\begin{aligned}
& \mathscr{A}_{2}>\mathscr{A}_{1}, \\
& \mathscr{A}_{2}>1+\frac{B \beta_{3}}{\left(d+\varepsilon_{2}\right)\left(d+\varepsilon_{3}\right)}, \\
& 1+\frac{\beta_{2}\left(d+\varepsilon_{3}\right)}{r_{3} \beta_{3} d} \geq \mathscr{A}_{1}+\frac{B \beta_{2}}{r_{3} d\left(d+\varepsilon_{2}\right)\left(\left(\mathscr{A}_{2} / \mathscr{A}_{1}\right)-1\right)},
\end{aligned}
$$

and these conditions refer to the failure of the treatment, as indicated by his name. The partial success equilibrium $E_{3}$ exists and is globally asymptotically stable if

$$
\begin{aligned}
& \mathscr{A}_{2}>\mathscr{A}_{1}+\frac{A B r_{1} \beta_{1} \beta_{3}}{d\left(d+\varepsilon_{1}\right)\left(d+\varepsilon_{2}\right)\left(d+\varepsilon_{3}\right)}, \\
& 1+\frac{\beta_{2}\left(d+\varepsilon_{3}\right)}{r_{3} \beta_{3} d}<\mathscr{A}_{1}+\frac{B \beta_{2}}{r_{3} d\left(d+\varepsilon_{2}\right)\left(\left(\mathscr{A}_{2} / \mathscr{A}_{1}\right)-1\right)} .
\end{aligned}
$$

These results indicate the partial success of $\mathrm{M} 1$ virus in decreasing tumor cells and increasing normal cells, which can reduce the tumor's size and stabilize the disease progression.

From the above analytical results, we remark that the Caputo fractional derivative's memory does not affect the stability analysis of equilibria. Based on the numerical simulations, we observe that the fractional order affects the speed of convergence and the time for arriving to equilibria (Figures 1-4).

The results obtained in this study are based on the fractional derivative in sense of Caputo with singular kernel. It will be more interesting to model the dynamics of oncolytic M1 virotherapy by using the new generalized fractional derivative with nonsingular kernel [10]. Moreover, we will extend our model presented in (1) by taking into account other biological factors such as diffusion $[11,12]$ and immunity $[13,14]$.

\section{Data Availability}

The data used to support the findings of this study are available from the corresponding author upon request.

\section{Conflicts of Interest}

The authors declare that they have no conflicts of interest.

\section{References}

[1] National Cancer Institute, "What is cancer?," 2019, https:// www.cancer.gov/about-cancer/understanding/what-iscancer.

[2] Cancer Research Institute, "How oncolytic virus therapy is changing cancer treatment?," 2012, https://www. cancerresearch.org/immunotherapy/treatment-types/ oncolytic-virus-therapy.

[3] Y. Lin, H. Zhang, J. Liang et al., "Identification and characterization of alphavirus M1 as a selective oncolytic virus targeting ZAP-defective human cancers," Proceedings of the National Academy of Sciences, vol. 111, no. 42, pp. E4504E4512, 2014.
[4] Z. Wang, Z. Guo, and H. Peng, "A mathematical model verifying potent oncolytic efficacy of M1 virus," Mathematical Biosciences, vol. 276, pp. 19-27, 2016.

[5] A. M. Elaiw, A. D. Hobiny, and A. D. Al Agha, "Global dynamics of reaction-diffusion oncolytic M1 virotherapy with immune response," Applied Mathematics and Computation, vol. 367, Article ID 124758, 2020.

[6] K. S. Cole, "Electric conductance of biological systems," Cold Spring Harbor Symposia on Quantitative Biology, vol. 1, pp. 107-116, 1933.

[7] A. Boukhouima, K. Hattaf, and N. Yousfi, "Dynamics of a fractional order HIV infection model with specific functional response and cure rate," International Journal of Differential Equations, vol. 2017, Article ID 8372140, 8 pages, 2017.

[8] C. Vargas-De-León, "Volterra-type Lyapunov functions for fractional-order epidemic systems," Communications in Nonlinear Science and Numerical Simulation, vol. 24, no. 1-3, pp. 75-85, 2015.

[9] J. Huo, H. Zhao, and L. Zhu, "The effect of vaccines on backward bifurcation in a fractional order HIV model," Nonlinear Analysis: Real World Applications, vol. 26, pp. 289-305, 2015.

[10] K. Hattaf, "A new generalized definition of fractional derivative with non-singular kernel," Computation, vol. 8, no. 2, pp. 1-9, 2020.

[11] K. Hattaf and N. Yousfi, "Global stability for fractional diffusion equations in biological systems," Complexity, vol. 2020, Article ID 5476842, 6 pages, 2020.

[12] A. M. Elaiw and A. D. Al Agha, "A reaction-diffusion model for oncolytic M1 virotherapy with distributed delays," The European Physical Journal Plus, vol. 135, no. 1, pp. 1-40, 2020.

[13] A. M. Elaiw and N. H. AlShamrani, "Analysis of a within-host HIV/HTLV-I co-infection model with immunity," Virus Research, vol. 295, Article ID 198204, 2021.

[14] K. Hattaf, "Global stability and Hopf bifurcation of a generalized viral infection model with multi-delays and humoral immunity," Physica A: Statistical Mechanics and Its Applications, vol. 545, Article ID 123689, 2020. 